\title{
Belgeo
}

Revue belge de géographie

$4 \mid 2016$

Sustainability of rural systems: balancing heritage and innovation

\section{Quelles trajectoires pour les territoires ruraux d'étangs en Lorraine (1970 à nos jours) ?}

Which trajectories for the rural areas of ponds in Lorraine?

\section{Denis Mathis}

\section{CpenEdition}

\section{Journals}

Édition électronique

URL : http://journals.openedition.org/belgeo/20254

DOI : $10.4000 /$ belgeo.20254

ISSN : 2294-9135

Éditeur :

National Committee of Geography of Belgium, Société Royale Belge de Géographie

Référence électronique

Denis Mathis, «Quelles trajectoires pour les territoires ruraux d'étangs en Lorraine (1970 à nos jours) ? », Belgeo [En ligne], 4 | 2016, mis en ligne le 19 septembre 2017, consulté le 01 mai 2019. URL : http:// journals.openedition.org/belgeo/20254; DOI : 10.4000/belgeo.20254

Ce document a été généré automatiquement le 1 mai 2019.

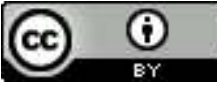

Belgeo est mis à disposition selon les termes de la licence Creative Commons Attribution 4.0 International. 


\title{
Quelles trajectoires pour les territoires ruraux d'étangs en Lorraine (1970 à nos jours) ?
}

Which trajectories for the rural areas of ponds in Lorraine?

\author{
Denis Mathis
}

1 Les pays d'étangs lorrains sont le plus souvent d'anciens systèmes productifs organisés autour de la carpiculture durant le Moyen-Âge. Occupant des espaces pionniers marginaux, ils se sont développés en appropriant l'hydromorphie des grandes dépressions argilo-marneuses ainsi que les versants ou les fonds de vallées du massif vosgien. Cette mise en valeur par la réalisation d'étangs a permis de développer une activité piscicole commerciale à destination des marchés urbains. Activité complémentaire à l'agriculture, elle a été organisée fréquemment en de grands domaines piscicoles détenus par les pouvoirs seigneuriaux. Un système productif fondé sur l'association de la carpiculture et de l'agriculture lors des assecs ou de la carpiculture et des prairies irriguées a permis de valoriser ces territoires. Cependant, à partir des XVIII ${ }^{\mathrm{e}}$ et XIX' siècles, de nouveaux usages de l'eau ont progressivement supplanté ce mariage de la « carpe et de l'épi » ou de la « carpe et du foin ». Les besoins de ressources en eau pour l'industrie, la régulation des canaux et le déclin de la pisciculture d'étangs ont progressivement entraîné l'étiolement de ces territoires ruraux peu productifs. L'exode rural a déclassé ces derniers entraînant leur mise en marge. Aujourd'hui, cette marginalité et sa permanence est fréquente tant à l'échelle nationale que régionale (Woessner, 2016; Mathis, 2016a ; Mathis, 2014).

2 Le dépérissement progressif de ces pays d'étangs a accentué la déprise agraire de ces territoires ruraux s'accompagnant d'un lent processus d'effacement des étangs. Cependant, et en comparant les pays d'étangs lorrains aux autres territoires d'étangs telles que la Dombes ou la Brenne, la construction identitaire de ces territoires d'eau est plus tardive ${ }^{1}$. Les communautés rurales ont souvent plus subi la présence et les contraintes des étangs qu'accepté ces derniers, synonymes de corvées et de perturbation 
des écoulements lors des vidanges. Ainsi, encore aujourd'hui cette question est régulièrement réactivée par le monde agricole lors des crues soulignant les enjeux des étangs au sein des hydrosystèmes. Par hydrosystème (Amoros, Petts, 1993), il faut comprendre " un système composé de l'eau et des milieux aquatiques associés dans un ensemble géographique délimité " (L. Touchart, 2005). De là, dérive la notion de limnosystème qui permet d'envisager l'étude croisée du plan d'eau et de son bassin d'alimentation. Cependant, cette limnicité, comme l'ont souligné P. Bartout et L. Touchart (2016), paraît insuffisante pour définir l'empreinte spatiale et sociétale des étangs et plans d'eau. Dans la logique particulière des pays d'étangs, il faudrait considérer un système stagnustre (un stagnosystème) conçu comme un anthroposystème (Levêque et al., 2003), c'est-à-dire un système interagissant entre deux ensembles constitués par un sociosystème et un écosystème considéré comme naturel/artificialisé. Ils évoluent avec le temps sous l'effet de facteurs externes et /ou internes au système.

3 Ainsi, cette notion permet de croiser étangs et territoires suivant une logique productive, culturelle, sociale, environnementale. Les territoires d'étangs lorrains - le Pays des Étangs, le Pays de l'Albe et des Lacs, la Woëvre, la Vôge, le Pays de Bitche... - hier déclassés, délaissés et marginalisés, s'inscrivent aujourd'hui dans des trajectoires nouvelles valorisant leur héritage stagnustre suivant des logiques et des cheminements multiples mais qui cherchent à établir des projets territoriaux soutenables, en valorisant leurs héritages et leur patrimoine paysager, environnemental, économique, tout en tenant compte des nouvelles pratiques habitantes. C'est cette évolution que nous allons présenter suivant une approche géohistorique et paysagère, afin de souligner les trajectoires de valorisation des pays d'étangs lorrains des années 1950 à nos jours. La marginalité agricole des pays d'étangs a permis d'offrir des opportunités car le patrimoine lentique est convoité. Cependant la valorisation des paysages d'étangs est établie en fonction de différentes lectures entre paysage nature et paysage ressource. Enfin, elle se traduit par des productions territoriales multiples qui traduisent des modèles soutenables de ruralités appelant à un usage raisonné des zones humides et inscrivant une gestion de la marginalité de ces territoires ruraux.

\section{Des paysages d'eau convoités}

\section{La singularité des pays d'étangs lorrains : les lacs-étangs}

4 Contrairement à de nombreux pays d'étangs telles que les Dombes ou la Brenne, les pays d'étangs lorrains sont caractérisés par la présence de gros étangs établis essentiellement sur les cours d'eau et s'organisant en chapelet ou en cascade. Lachaussée (360 ha), Bischwald (210 ha), Mittersheim (225 ha), Lindre (622 ha), le Stock (750 ha), Gondrexange (770 ha) constituent le cœur d'anciens domaines piscicoles organisés autour de ces « lacsétangs ». Malgré sa surface imposante le lac de Madine (1100 ha) doit être classé parmi cette catégorie des lacs-étangs de Lorraine. Ils modifient la perception de territoires d'eau et la notion de limnicité. Ainsi pour la Woëvre $\left(1500 \mathrm{~km}^{2}\right)$, la limnicité est de l'ordre de $8 \%$ couvrant 12000 hectares pour seulement une centaine d'étangs. Pour des surfaces en eau similaires, les Dombes $\left(1000 \mathrm{~km}^{2}\right)$ recensent 1100 étangs. Ce faible nombre d'étangs s'oppose à l'importance des surfaces en eau modifiant sensiblement la place des étangs au sein du paysage. On perçoit difficilement une ambiance stagnustre, les étangs sont le plus souvent à l'écart des villages, ces derniers occupant les croupes interfluviales. En position 
périphérique au sein des terroirs, les étangs sont souvent bordés par les forêts masquant partiellement ces derniers.

Quelques grands domaines piscicoles ont échappé au démantèlement et au déclin maintenant une activité centrée sur la pêche accompagnée désormais d'activités pédagogiques ou de préservation. Ainsi le Domaine du Lindre ou le Domaine du Vieux Moulin à Lachaussée perpétuent une activité de pisciculture extensive et de commercialisation. Les autres lacs-étangs ont vu leurs ressources en eau remobilisées notamment pour régulariser les débits des canaux. Ainsi au XIX ${ }^{e}$ siècle, lors de la mise en eau du canal de la Marne au Rhin et puis du canal des Houillères, les étangs du Stock, de Gondrexange ou de Mittersheim ne sont plus vidangés et pêchés. Avec le déclin des activités piscicoles et agricoles, les grands étangs bordés de forêts constituent un " paysage héritage » mais c'est également un «paysage ressource» convoité pour des activités de loisirs. Cette appétence est liée à la valeur réelle et symbolique de ces paysages d'eau.

Figure 1. Les pays d'étangs lorrains.

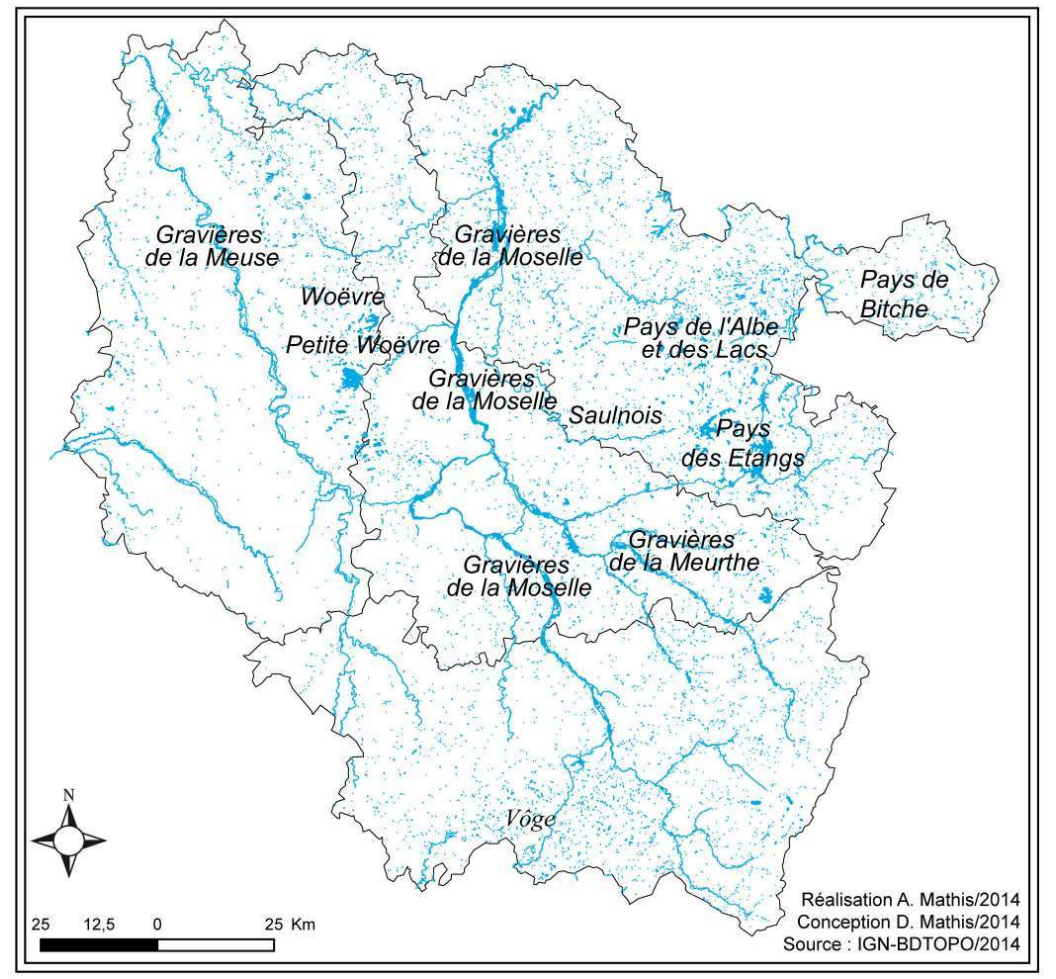

Source : Mathis et Mathis, 2016

La Lorraine présente un riche patrimoine lentique. Cependant, ses pays d'étangs se sont souvent imposés tardivement au sein des paysages agraires lorrains.

\section{La reconquête pionnière des étangs}

6 Le déclin des activités agricoles dans les pays d'étangs a fait naître des opportunités liées à la faiblesse des coûts du foncier des parcelles de prairies bordant les étangs. L'acquisition des espaces rivulaires individuellement ou collectivement s'est traduite par un processus de «cabanisation » multiforme. À l'origine, il s'agit d'habitations légères de loisirs, de pontons de pêches, de campings ou de formes de copropriété-lotissements, 
voire de lotissements dont l'installation pionnière s'est progressivement indurée. Elles évoluent vers une consolidation des implantations autour souvent de caravanes ou de bungalows désormais sédentarisés. La pérennisation des formes d'habitats se traduit par leur consolidation par de multiples ajouts désormais «en dur». Auvents, terrasses, chalets complètent ces résidences secondaires. Cependant le rythme d'évolution de ce type d'habitat de vacances et de week-end souligne la capacité d'attraction des étangs ( $72 \%$ des logements des communes riveraines de l'étang du Stock sont des résidences secondaires et des logements occasionnels), qui s'inscrit également par une relative émulation entre résidents. Si certaines de ces copropriétés ont été dissoutes car trop complexes à gérer, c'est souvent pour prendre des formes plus indurées telles que des «villages» de chalets, voire de maisons individuelles du type pavillons. Ce processus d'urbanisation désordonnée, souvent en marge des règles d'urbanisme, a élaboré un paysage rivulaire décrié, notamment par le Parc Naturel Régional de Lorraine (PNRL). Ce discrédit est lié à l'opposition entre la politique de préservation des étangs notamment dans sa démarche de « sauvetage » du Domaine du Lindre et la volonté des municipalités d'accepter un développement touristique désordonné. Cependant dans le contexte d'abandon des vieux noyaux villageois, se traduisant par la dédensification structurelle avec l'apparition de "dents creuses », le dynamisme de ces copropriétés et lotissements touristiques avec une population nouvelle souvent allemande a accentué le sentiment de déclassement.

7 Depuis la conquête pionnière des berges des étangs, le processus de cabanisation est désormais accompagné et en voie de normalisation notamment en terme d'assainissement par exemple, ce qui souligne une évolution raisonnée et progressive de l'occupation des berges des étangs. De l'opportunité foncière est née une transformation des paysages d'étangs dont la valorisation en espace touristique et de détente évolue vers un espace résidentiel durable qui s'intègre à l'espace rural, revivifiant ce dernier par une perméabilité entre lotissements touristiques et anciens noyaux villageois. Cette symbiose est la plus aboutie autour de l'étang du Stock comme l'a souligné D. Mathis (2014). Ce modèle pourtant combattu par les acteurs du PNRL rencontre aujourd'hui un soutien politique plus affirmé.

8 Ces paysages et territoires d'eau convoités ont permis de développer, comme le souligne plus généralement Y. Le Caro (2012) pour les territoires ruraux, des formes d'assolement touristique et récréatif. Les pays d'étangs ont des paysages qui peuvent être qualifiés d'authentiques et c'est cette authenticité qui est convoitée (Luginbühl, 2011). Au sein d'un espace agricole en évolution et/ou les spécificités et les identités s'estompent, les paysages d'eau apparaissent comme immobiles, voire immuables, il s'agit donc d'un paysage ressource.

Le regard porté sur la valeur écologique des étangs reste ambivalent. Ils sont vus comme un élément perturbateur des écoulements et des hydrosystèmes, aussi leur effacement est préconisé surtout lorsqu'ils sont « installés au fil de l'eau », accusés d'être un obstacle à la bonne gestion environnementale et écologique, notamment en ce qui concerne la qualité des eaux, voire dans la gestion des écoulements particulièrement lors des épisodes de crues à l'aval des étangs. Cependant dans l'espace lorrain, les grands lacs-étangs sont présentés comme des facteurs de biodiversité. Il suffit de constater l'empilement des formes de protection et de préservation mises en œuvre autour de ces derniers (Mathis et Mathis, 2013; Chiffre et Mathis, 2015) pour comprendre l'importance qui leur est accordée. Cette superposition de mesures diverses de labels de protection et d'inventaires 
(RAMSAR, loi Littoral, ZNIEFF...) organise une «mise sous cloche $»^{2}$. Il faut cependant nuancer le propos concernant les étangs de tailles plus réduites particulièrement ceux organisés en cascade. Ainsi, la politique de restauration des berges, d'entretien et de renaturation des étangs observables dans et hors du PNRL s'oppose à des politiques d'effacement des étangs notamment dans le Parc Naturel des Vosges du Nord suivant une logique de rétablissement des continuités écologiques.

\section{Des étangs au cœur de projets touristiques et de loisirs}

\section{De multiples formes d'assolement touristique}

10 Les démarches pionnières initiées depuis les années 1960 sont aujourd'hui pérennes. De nombreux pôles se sont consolidés, ils forment des lotissements de résidences secondaires voire pour les formes les plus abouties des résidences secondes expression de nouvelles pratiques habitantes, le plus souvent entre le Land de Sarre (Allemagne) et les pays d'étangs mosellans (Mathis, 2014 ; Mathis et Mathis, 2016). Les activités nautiques et de loisirs se sont structurées (yacht club, bateau d'excursion, tourisme fluvial sur les canaux déclassés de la Marne au Rhin, de l'Est ou des Houillières). Un maillage d'activités touristiques a vu le jour. Certes dans le Saulnois, l'expérience de la Route gastronomique des poissons a échoué et la Maison du Pays des Étangs à Tarquimpol reste confidentielle. En revanche, le Domaine du Lindre à Lindre-Basse ou encore le Parc animalier de SainteCroix sont de belles réalisations d'appel autour de la valorisation des étangs et de la découverte de la nature. Avec 310000 entrées en $2015^{3}$, c'est aujourd'hui un des acteurs incontournables de la valorisation de la région. À une autre échelle, le Domaine du Lindre totalise 47000 visites en 2015 notamment dans le cadre des journées des Grandes Pêches qui voient affluer sur site de 12000 à 13000 visiteurs. Le paysage des étangs est une ressource s'inscrivant dans un « récit » à base de nature, de biodiversité et d'érème.

11 Le label Ramsar permet d'afficher le domaine au salon international du tourisme ornithologique européen British Bird Watching Fair. C'est sur ces thématiques que se développent les nouveaux usages de l'eau. Ainsi dans le Pays des Étangs, les villages de Rhodes et Langatte ont connu de nettes reprises démographiques ces dernières années, justifiant un déploiement de nouveaux services pour une population rurale et de touristes. Cependant, la soutenabilité de ce modèle porté par 4 communes de petites tailles peut-être posée. La réussite de ce pôle touristique et de loisirs doit désormais trouver de réels relais au sein d'un territoire plus vaste, avoir le soutien des acteurs touristiques départementaux et régionaux. La question est toujours de pouvoir concevoir des espaces transitionnels entre vieux noyaux villageois et nouveaux lotissements. Les villages ont trouvé un relais d'activité, des formes de redynamisations rurales se mettent en place. Ainsi, les maisons lorraines retrouvent une attractivité, elles sont modernisées, perdant parfois leur caractère rural par la transformation des travées de granges et d'écurie-étables. Cependant, la préservation d'une partie du bâti permet de conserver les éléments les plus remarquables, notamment pour des formes d'habitats les plus traditionnels comme l'habitat à pan de bois dans l'Est de la Lorraine. La valorisation du patrimoine vernaculaire (puits à balanciers, fontaines, guéoirs ${ }^{4} .$. ) accompagne un lent processus de transformation des villages dont la structure reste héritée du système agricole du village lorrain, mais qui s'intègre désormais aux nouvelles formes de la 
ruralité marquée par des pendularités semaine/week-end voire par des "habiter » de résidences secondes. L'ancienne coquille vide du village retrouve une consistance en s'adaptant à ces nouvelles pratiques habitantes et à l'arrivée de nouveaux habitants. Ces derniers, par leur demande, stimulent les municipalités dans les réponses à établir pour la restructuration de l'espace public. Il ne faut pas oublier que la conquête collective des berges par des lotissements, des campings ou des pontons/cabanes de pêche restent une forme collective d'usage des étangs. Les villages doivent se tourner désormais vers les berges, les aménager et les conquérir alors qu'historiquement elles les délaissaient. Ainsi nait en Lorraine une société des étangs construite, non sur la pisciculture et l'agriculture, mais sur l'usage résidentiel des berges des étangs.

Figure 2. Autour de la valorisation du paysage ressource.

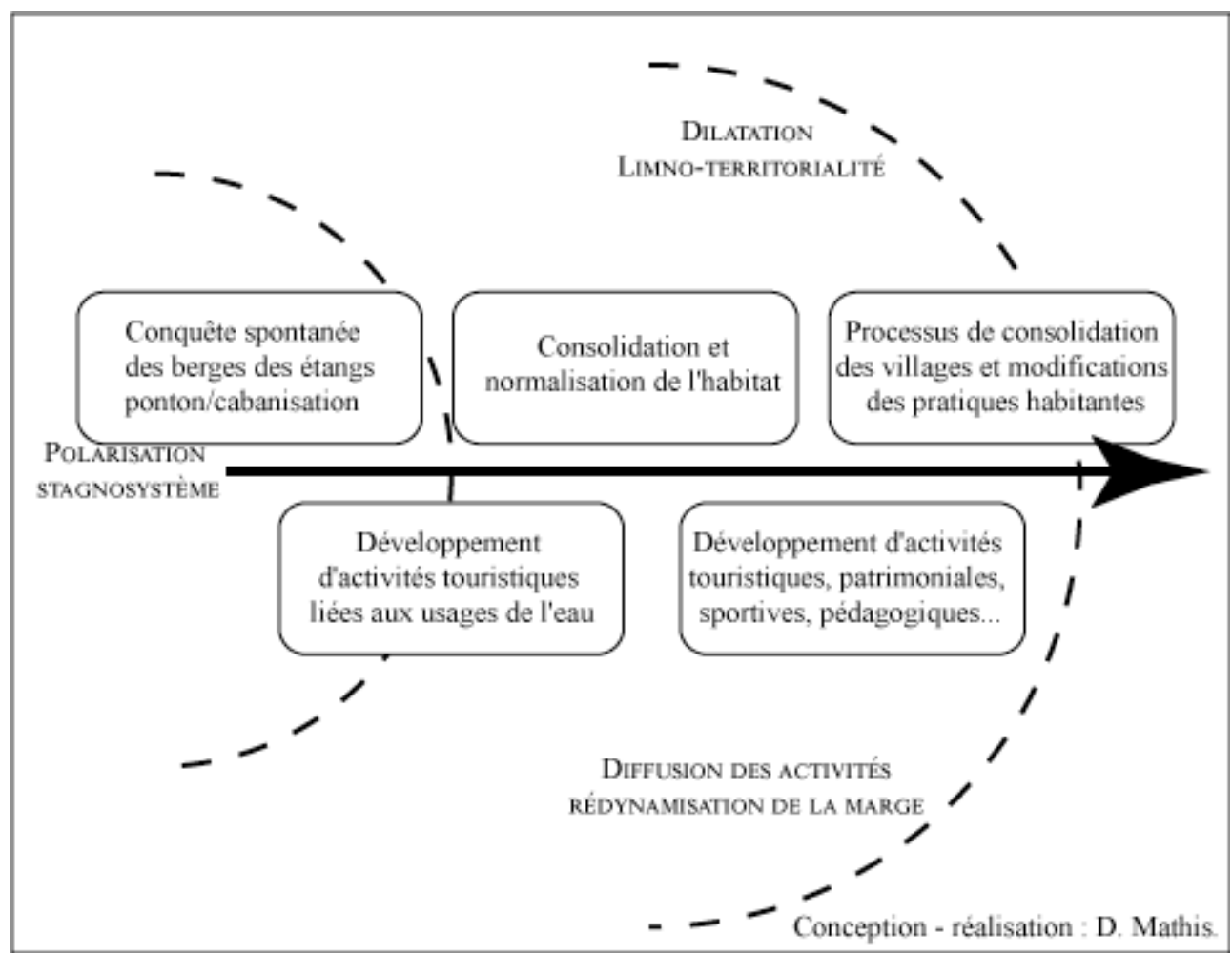

La ressource constituée par les étangs est un atout dans la reconquête des espaces ruraux fragilisés. Paysage convoité, revalorisé par l'activité touristique et de loisirs, sa mise en scène permet de construire un territoire lentique.

Ce modèle est une forme collective qui s'oppose au modèle de développement des étangs d'agréments observable dans les Vosges du Nord, dans les Mille Étangs, dans la Vôge ou dans les vallées du massif vosgien. Cette appropriation des étangs traduit un modèle de privatisation et de nucléarisation du patrimoine lentique. Ce phénomène de colonisation/ cabanisation se retrouve également pour les anciennes gravières reconverties (Mathis, Chiffre \& Harmand, 2015 ; Mathis et Mathis, 2016). Ces nouveaux étangs ont des formes de valorisation similaires aux vieux pays d'étangs entre la promotion d'agrément, la mise en œuvre de pontons/cabanes... mais aussi désormais dans la mise en œuvre de programmes écologiques notamment en lien avec les corridors des trames vertes et bleues.

Aujourd'hui, la question du tourisme dépasse la gestion de la cabanisation des berges, c'est-à-dire une mise aux normes d'un processus de conquête désordonnée des berges. 
Dans le Pays de l'Albe et des Lacs, où les étangs militaires ont été établis dans le cadre de la mise en œuvre de la Ligne Maginot aquatique, ces derniers ont permis une construction territoriale. En mars 1971, la commune de Puttelange-lès-Farschviller, au cœur de ce territoire, est devenue Puttelange-aux-Lacs, amorçant l'appropriation du patrimoine lentique qui donne ensuite naissance au Pays de l'Albe et des Lacs. Les étangs délaissés par l'armée ont fait l'objet d'une colonisation des berges dans les années 1970. Le processus d'induration au sein du paysage rivulaire et son impact sur la qualité des eaux a conduit à des travaux de renaturation et de restauration des hydrosystèmes conciliant aujourd'hui valorisation touristique et de loisirs et gestion raisonnée de la ressource en eau et des paysages stagnustres (Chiffre et Mathis, 2013). La renaturation des cours d'eau notamment du Mutterbach n'a pas impacté le paysage d'étangs et s'est fait dans une logique de valorisation de l'héritage militaire destinée à renforcer, poursuivre et diversifier l'attractivité de ce territoire autour du tourisme de mémoire. Cette dilatation permet de construire une offre touristique élargie destinée à lentement irriguer les territoires. Ce territoire offre finalement l'exemple d'une limno-territorialité construite sur les étangs et l'usage de l'eau.

\section{La tentation de la « mise sous cloche»}

14 Toutefois, cette trajectoire pionnière des étangs qui repose sur une forme prédatrice du foncier agricole en déprise s'est heurtée aux enjeux environnementaux des étangs. Cette approche a été particulièrement développée par le PNRL. La démarche s'inscrit dans une lecture du paysage patrimoine naturel dont il faut garantir la préservation de l'héritage lentique face à l'urbanisation touristique. Elle a trouvé des soutiens auprès des départements de la Meuse et de la Moselle qui ont relayé cette approche, notamment lorsque le département de la Moselle s'est porté acquéreur du Domaine du Lindre en 1976. Comme l'ont souligné E. Chiffre et D. Mathis (2015), l'action du PNRL a été déterminante dans la prise en compte de la qualité environnementale et écologique des hydrosystèmes et plus particulièrement des étangs. En associant de multiples acteurs, le PNRL a réussi à établir une démarche de protection des centres de piscicultures en relayant ces derniers par des projets de valorisation, associant autour de la patrimonialisation de pisciculture des activités de loisirs, tout en assurant la préservation des berges. C'est dans cette logique qu'il faut relever les "Grandes Pêches ", moment de promotion qui permet en un week-end de faire venir sur site de nombreux visiteurs (Mathis et Chiffre, 2015). Ainsi, pour le Domaine du Lindre c'est entre 11000 et 13000 personnes accueillies lors des grandes pêches, 40000 à 45000 visiteurs par an. La valorisation de cette activité permet de conforter ce patrimoine et complète la notion de limnicité (De Carraras, Le Lay, 2016; Bartout, 2015 ; Bartout, Touchart, 2016). L'empilement des mesures de protection au niveau de ces étangs constitue une des principales caractéristiques comme l'ont souligné D. Mathis et A. Mathis (2013) pour le Domaine du Lindre ${ }^{5}$. La question concerne finalement plus la lecture dans le contexte local que l'accumulation et l'empilement de ces mesures aux périmètres variables. Ainsi, la démarche a longtemps eu pour objectif de sanctuariser certains étangs en ajoutant des contraintes (Mathis, 2013). 


\section{Approche sensible du paysage des étangs} préservation environnementale, la biodiversité et les activités récréatives et de loisirs, porteuses d'avenir. Pour ces systèmes ruraux, les trajectoires reposent sur des compromis entre paysage nature et paysage ressource.

La logique d'inscrire les étangs lorrains dans une démarche centrée sur la préservation et la conservation d'un patrimoine paysager naturel permet de favoriser un modèle où l'environnement est au cœur d'une démarche qu'il convient de valoriser, d'expliciter. Que ce soit à Lachaussée ou au Lindre, les enjeux sont d'abord d'assurer la gestion des étangs, leur qualité paysagère, de pérenniser le fonctionnement des étangs et d'une pisciculture en associant un public à cette démarche sans modifier sensiblement les territoires. L'enjeu n'est pas de faire sortir ce terroir de sa marginalité, mais de préserver ce paysage et son activité en relayant les choix par des activités de protection des paysages, de l'environnement, d'assurer autour de ce projet une sensibilisation à la protection de l'environnement par des démarches pédagogiques, de maintenir également une activité piscicole dont les deux domaines sont les pôles principaux faits d'héritages et de patrimonialisation des usages. Aujourd'hui, s'agrège à ce modèle la promotion de circuits alimentaires courts associés aux activités évènementielles et pédagogiques (Mathis, Chiffre, 2015). Le modèle s'est diffusé en marge des politiques menées par les parcs naturels régionaux ; la filière piscicole extensive est devenue innovante, développant des formules d'aquatourisme qui bien qu'encore artisanales prolongent l'idée que l'étang n'est pas seulement un espace productif. Cette logique territoriale trouve des relais auprès des conseils généraux et régionaux et s'est organisée derrière la Filière Lorraine d'Aquaculture Continentale (FLAC). À ce jour, la Lorraine produit 1100 tonnes de poissons sur 7000 ha de plans d'eau. Cependant l'avenir d'une partie des étangs reste conditionné aux enjeux de continuité écologique et à la Directive Cadre Européenne sur l'Eau 2000 (Millot, 2016). Faire comprendre la valeur patrimoniale d'un paysage mais aussi d'un savoir-faire permet d'inscrire la limnicité dans une logique plus complexe. Aujourd'hui, les producteurs s'inscrivent dans des démarches pédagogiques notamment par l'accueil de scolaires soit dans une démarche touristique destinée à faire connaître tout autant qu'à diversifier l'activité piscicole. En cela, il s'agit d'une duplication à une moindre échelle du modèle développé autour du Domaine du Lindre ou du domaine de Lachaussée. Bien qu'encore balbutiante, la démarche présente une valorisation soutenable construite sur une lecture du paysage d'étangs tournée sur la nature et la gestion de la ressource en eau. Toutefois, la démarche protectrice peut parfois être lue comme exclusive. Le paysage des étangs bien que riche reste somme toute ordinaire. Le préserver induit un paysage élitiste destiné à quelques-uns dans une logique méritante d'une démarche touristique et considérant les territoires ruraux comme des réserves. Cependant cette approche permet de conforter la valeur environnementale mais aussi d'inscrire les étangs dans des démarches patrimonialisantes. 
Figure 3. Autour de la préservation du paysage nature.

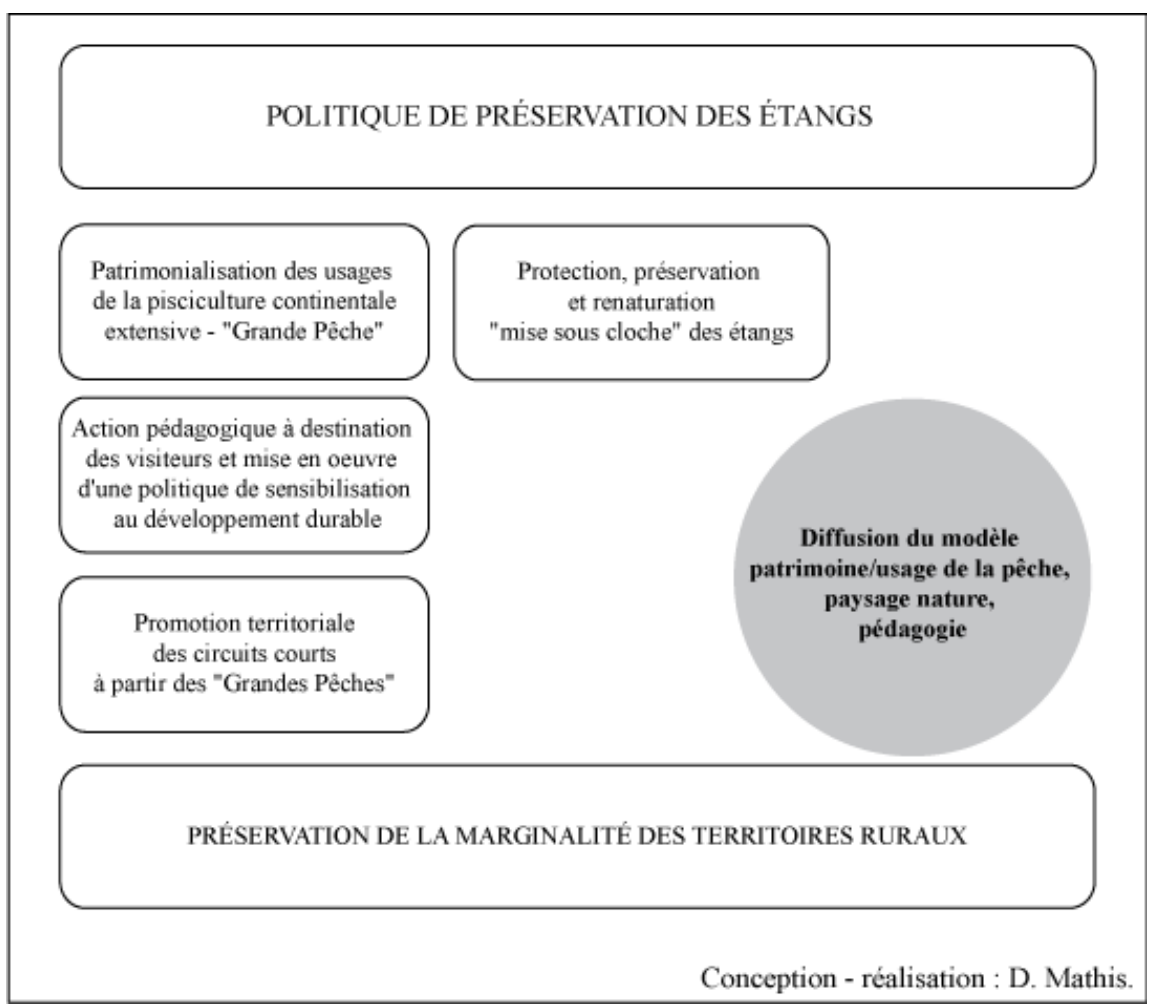

La politique de préservation du patrimoine environnemental des étangs permet de développer un modèle touristique moins impactant et plus raisonné, ménageant ressource et milieu.

Il faut en cela reconnaître le rôle pionnier des parcs naturels régionaux et particulièrement du PNRL dans la mise en œuvre de protection /préservation des étangs et la valorisation d'un paysage nature. Cependant la question est de savoir si ces derniers n'ont pas parfois une approche trop exclusive. En ostracisant le modèle de l'étang du Stock, le PNRL a mis en œuvre une politique tournée vers la protection et la préservation environnementales des étangs, oubliant parfois la nécessité de développer une activité touristique mais aussi en bridant les processus d'affirmations et recompositions territoriales pour le sud-mosellan. Sans les capacités d'accueil touristique de la zone de l'étang du Stock, l'activité touristique des étangs trouve sa place dans l'accès à l'étang. Un des enjeux de la durabilité des territoires ruraux d'étangs revient à décentrer les points de vue, d'aborder une démarche conciliante entre la mise sous cloche environnementale et les pratiques habitantes nouvelles. Donner une chance à la marginalité lorsque celle-ci permet d'entrer dans des processus de redynamisation territoriale. Faut-il réduire les territoires ruraux marginaux pour assurer les îlots et les corridors écologiques à l'échelle des territoires régionaux? Une politique raisonnée de l'eau et de sa gestion doit permettre de concilier les diverses approches afin de construire un projet collectif qui d'un point de vue opératoire valorise le paysage des étangs comme une ressource en considérant celui-ci comme un héritage dont la valeur environnementale est imparfaite mais qu'il est nécessaire de préserver et de protéger tout en considérant que ce milieu artificiel doit trouver une fonctionnalité, ce en quoi les démarches pédagogiques sont tout aussi importantes que les démarches environnementales. Le paysage des étangs ne doit pas être traité comme un paysage relique qu'il faut impérativement fossiliser. La question de l'urbanisation des rives des étangs longtemps perçue comme consommatrice 
d'espace "naturel» a en fait polarisé sur certaines berges l'habitat, laissant libres certaines " cornées ». Si on compare à l'urbanisation des lacs en Finlande, ou simplement du Pays des Mille Étangs en Bourgogne-Franche Comté (Mathis, 2016b), l'urbanisation densifiée des berges est certainement plus soutenable que la gestion dispersée et morcelée. La question n'est pas d'opposer les formes d'urbanisation entre le village traditionnel et les villages de résidences secondaires/secondes mais de voir comment construire une cohérence et une unité nouvelle au sein du territoire d'étangs en repensant la ruralité des pays d'étangs.

\section{Conclusion}

Aujourd'hui, les territoires d'étangs lorrains sont en recomposition, convoités par une multitude d'acteurs (propriétaires d'étangs privés, communes, départements), leurs trajectoires sont multiples. Ces territoires sont au cœur d'enjeux d'aménagement ou de projets territoriaux. L'héritage paysager de ces anciens territoires de la pisciculture continentale évolue. Désormais deux trajectoires semblent se structurer: l'une autour d'un territoire construit sur la logique d'un "paysage nature » et l'autre sur une logique d'un «paysage ressource». Les productions territoriales qui en résultent dépassent le cadre des étangs et concernent une enveloppe spatiale amenée à se dilater. Les deux logiques combinent à des degrés divers, activités de loisirs et de détente, logiques de protection de préservation de la biodiversité, activités pédagogiques. La combinaison des trois modifie les pratiques habitantes des territoires ruraux. Si les parcs naturels ont été des acteurs importants des politiques de gestion des zones humides et particulièrement des étangs la gestion des stagnosystèmes est aussi l'œuvre d'une multitude d'acteurs locaux notamment les propriétaires d'étangs. Aujourd'hui, pour tous ces acteurs il y a la volonté de trouver un équilibre entre nouvelles pratiques habitantes, fréquentation touristique et de loisirs, pédagogie autour de la gestion et de la préservation des zones humides, patrimonialisation des usages et/ou réhabilitation des étangs. 
Figure 4. Deux trajectoires des territoires d'étangs lorrains.

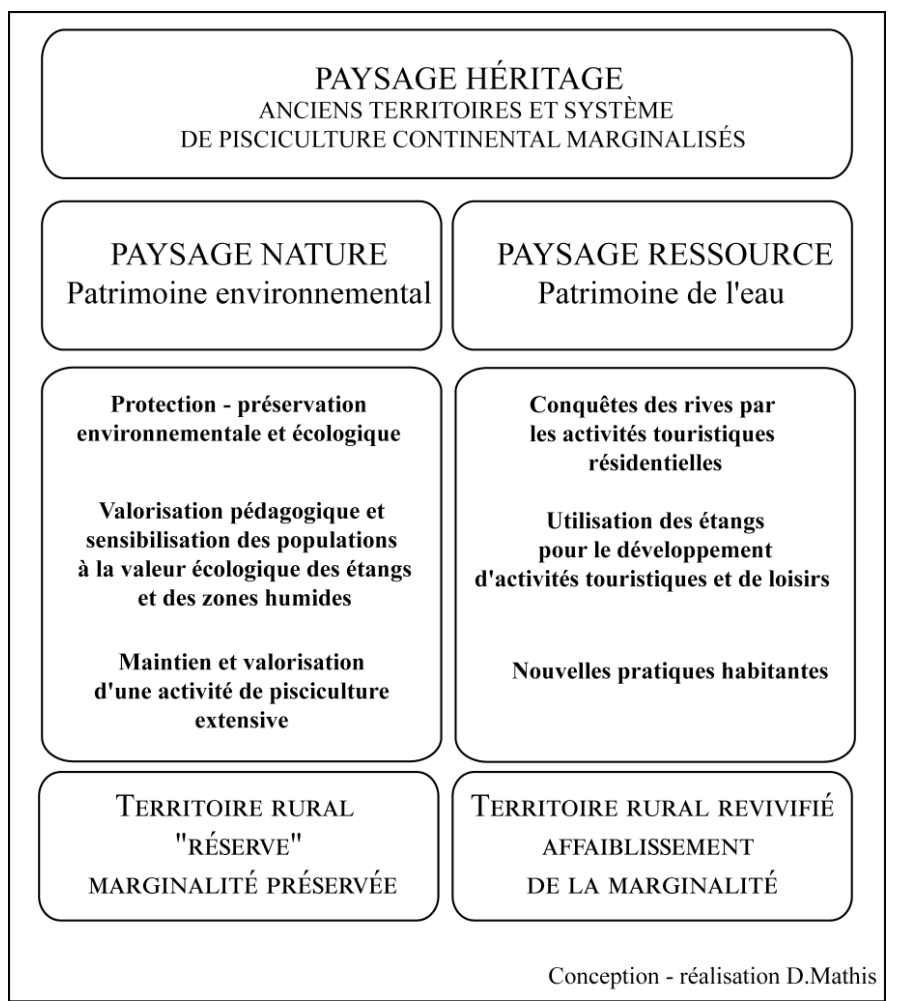

À partir d'un héritage stagnustre peu valorisé, deux trajectoires ont été développées : une trajectoire « environnementale », une trajectoire « ressource».

La soutenabilité des modèles réside également dans leur capacité à irriguer durablement les territoires ruraux afin de faire sortir ces derniers de leur marginalité et du déclin rural. Trouver des relais autour des pôles des étangs, développer les activités d'aquatourisme, assurer des mises en réseau, la nouvelle région Grand Est peut à ce titre constituer un atout dans une redéfinition des projets coopératifs autour de territoires ayant des enjeux patrimoniaux communs (Lac du Der, Sundgau...).

\section{BIBLIOGRAPHIE}

AMOROS C., PETTS G.E. (1993), Hydrosystèmes fluviaux, Collection Écologie, no 24, Masson, 300 p.

BARTOUT P. (2015), Les territoires limniques. Nouveau concept limnologique pour une gestion géographique des milieux lentiques, mémoire HDR de géographie, Université d'Orléans, 444 p.

BARTOUT P. (2016), « Le territoire comme palimpseste lentique : une vision spatiale géohistorique des interactions hommes-milieux-plans d'eau ", Revue Géographique de l'Est, 56, 1-2, http://rge.revues.org/5723. 
BARTOUT P., TOUCHART L. (2016), « La notion de limnicité est-elle suffisante pour caractériser l'empreinte spatiale et sociétale des plans d'eau? ", Revue Géographique de l'Est, 56, 1-2, http:// rge.revues.org/5766.

BÉDOUCHA G. (2011), Les liens de l'eau. En Brenne, une société autour de ses étangs, Paris, co-édition QUÆ Éditions et Editions de la Maison des sciences de l'homme, collection Natures sociales, 688 p.

CHIFFRE E., MATHIS D. (2015), « Prise en compte des hydrosystèmes hérités par les parcs naturels régionaux de Lorraine et du Morvan ", Territoire en mouvement, Revue de géographie et aménagement, 25-26, http://tem.revues.org/2764.

DE CARRARA S., LE LAY Y.F. (2016), « Quand l'usage fait patrimoine. Vers une patrimonialisation des usages et des paysages culturels ? », Développement durable et territoires, 6, 1, mars 2015, http:// developpementdurable.revues.org/10754.

LE CARO Y. (2012), « Les agriculteurs et le partage de l'espace agricole pour des usages récréatifs », in PAPY F., MATHIEU N. \& FÉRAULT Ch. (dir.), Nouveaux rapports à la nature dans les campagnes, Éditions QUÆ, pp. 101-117.

LÉVÊQUE Ch., MUXART T., ABBADIE L., WEIL A. \& van der LEEUW S. (2003), « L'anthroposystème : entité structurelle et fonctionnelle des interactions sociétés-milieux ", in LÉVÊQUE Ch., van der LEEUW S. (éd.), Quelles natures voulons-nous ?, Elsevier, Paris, pp. 110-129.

LUGINBÜHL Y. (2011), « Un paysage authentique », Cahiers du musée des Confluences, pp. 45-57.

MATHIS D. (2014), « Recomposition territoriale d'un espace rural flou, L'exemple du "Pays des Étangs" en Moselle », Territoire en mouvement, Revue de géographie et aménagement, http:// tem.revues.org/2415.

MATHIS D., CHIFFRE E. \& HARMAND D. (2015), « Les enjeux paysagers de la reconversion en étangs des sablières et gravières ", in TOUCHART L., BARTOUT P. \& MOTCHALOVA O. (dir.), Mieux comprendre les étangs. Expériences nationales et internationales, Brive, Éditions « les Monédières », pp. 383-385.

MATHIS D., CHIFFRE E. (2015), « Les grandes pêches : traditions renouvelées », in TOUCHART L., BARTOUT P. \& MOTCHALOVA O. (dir.), Mieux comprendre les étangs. Expériences nationales et internationales, Brive, Éditions « les Monédières », pp. 389-391.

MATHIS D., MATHIS A., (2016), « Anciens et nouveaux terroirs d'étangs en Lorraine », Revue Géographique de l'Est, 56, http://rge.revues.org/5712.

MILLOT C. (2016), « Incidences de la notion de cours d'eau sur la gestion et la pérennité des étangs. Le cas de la dérivation », Revue Géographique de l'Est, 56, 1-2, http://rge.revues.org/5803.

TOUCHART L. (2005), « Hydrosystème », Hypergéo, http://www.hypergeo.eu/spip.php?article313.

TOUCHART L., BARTOUT P. \& MOTCHALOVA O. (dir.) (2015), Mieux comprendre les étangs. Expériences nationales et internationales, Brive, Éditions « les Monédières », 421 p.

WOESSNER R. (2016), « La Route de la Carpe frite dans le Sundgau : enjeu identitaire, enjeu territorial », Revue Géographique de l'Est, 56, 1-2, http://rge.revues.org/5786.

\section{NOTES}

1. Pour le pays de Bitche ou la Woëvre, la spécificité des étangs n'a jamais été un marqueur territorial historique. Quant à l'appellation de « Pays des Étangs », elle 
n'apparaît que progressivement à partir du XIX ${ }^{\mathrm{e}}$ siècle par affaiblissement des vieux pays lorrains périphériques (Mathis, 2014).

2. Cette mise sous cloche est particulièrement perceptible au sein du Domaine du Lindre avec 6 périmètres différents de protection et d'inventaires.

3. Les sites - Les événements, Moselle 2015, http://www.moselle-tourisme.com/images/ fichiers/Fequentation\%20des\%20sites\%20en\%202015.pdf.

4. Guéoir ou égayoir (Lorraine) : petite mare empierrée aménagée en pente douce et destinée à baigner les chevaux.

5. En 2017, projet d'extension de la zone Ramsar sur la partie est du PNRL, élaboration d'un dossier de candidature au label Réserve Mondiale de Biosphère (Sarrebourg).

\section{RÉSUMÉS}

Cet article envisage les dynamiques des territoires ruraux d'étangs en Lorraine. La fragilité et la marginalité de ces territoires ruraux ont permis de construire autour des pôles des étangs deux trajectoires différentes: la protection et la valorisation. Construites en grande partie sur une lecture patrimoniale du paysage d'étangs, elles illustrent des choix de projets territoriaux fondés sur de nouveaux assolements. Les enjeux des pays d'étangs résident aujourd'hui dans leur capacité à gérer un paysage héritage et d'assurer finalement la gestion de ce paysage nature ou de ce paysage ressource.

This article considers the dynamics of the rural territories of ponds in Lorraine. The fragility and the marginality of these rural areas build two different trajectories around the poles of the pond. The reading of these landscapes illustrates the choices of new rotation-based territorial projects. Nowadays, the issues in the country of ponds lie in their ability to manage a heritage landscape and ultimately ensure the management of this landscape nature or landscape resource.

\section{INDEX}

Mots-clés : étangs, paysage nature, paysage ressource, marginalité, Lorraine, France

Keywords : ponds, landscape nature, landscape resource, marginality, Lorraine, France

\section{AUTEUR}

\section{DENIS MATHIS}

Laboratoire d'Observation des Territoires (LOTERR), Université de Lorraine, Nancy, denis.mathis@univ-lorraine.fr 